\title{
Magneto-Ionic Propagation in Inhomogeneous Media. Part I. Transverse Propagation
}

\author{
B. K. Banerjea \\ Institute of Armament Technology, Poona, India
}

(Received March 29, 1965)

\begin{abstract}
Propagation of electromagnetic waves in an inhomogeneous magneto-ionic medium has been discussed with special reference to ionospheric propagation. This involves the study of two coupled linear second-order differential equations with coefficients having one essential and more than one regular singularity. The problem has been tackled in two stages, divided into parts I and II of this paper.

In part I, dealing with the transverse propagation in a parabolic electron layer, the wave equation belonging to a class higher than the confluent hypergeometric or Mathieu equations has been investigated. In part II, dealing with the oblique propagation, the effect of the coupling function for the above class of equations has been studied, and relevant asymptotic solutions for the coupled equations derived.

Mathematical expressions for the physical observables in ionospheric investigations have been derived from the solutions of the wave equations inside a parabolic electron layer.
\end{abstract}

\section{Introduction}

A partially ionized medium traversed by a steady magnetic field is termed a magneto-ionic medium; the ionosphere is such a medium, in which the electron density is a function of the vertical height. It can be shown from the Maxwell-Lorentz equations that the propagation of electromagnetic (em) waves inside such a medium is controlled by the equation

$$
\left[\frac{\partial}{\partial t}+\nu+\omega_{H} x\right]\left[\boldsymbol{\nabla} \times \boldsymbol{\nabla} \times \mathbf{E}+\mu_{0} \epsilon_{0} \frac{\partial^{2} \mathbf{E}}{\partial t^{2}}\right]+\frac{\mu_{0} N e^{2}}{m} \frac{\partial \mathbf{E}}{\partial t}=0,
$$

where mks units and symbols (see appendix) have been used.

The standard pulse technique method used for the study of ionospheric propagation furnishes information about the following observables:

(a) The interval $\tau$ taken by the pulse to travel from the ground to the ion layer and back.

(b) The fraction $R^{2}$ of energy (reflection coefficient) reflected back by the ion layer.

(c) The state of polarization of the downcoming wave as determined by the ratio $R$ of the polarization ellipse and the tilt angle $\Psi$ of the major axis with respect to the local magnetic meridian.

Any method developed for the study of magneto-ionic propagation of em waves must succeed in furnishing mathematical expressions in terms of the known parameters for these observables from the solutions of (1).

The ray optics of Appleton [1927] and Hartree [1929] form a special solution of (1) with the assumptions:

(i) $\frac{\partial}{\partial t}=2 \pi i f$, where $f$ is the frequency of the incident wave, and

(ii) $N=N_{0}$, a constant.

The observed values of $\tau$ are utilized in the ray optics for ascertaining the electron density $N$ as a function of the equivalent height $P^{\prime}=\frac{1}{2} c \tau$, a distance which light would have covered in an interval of $\tau / 2$. For frequencies less than the plasma frequency of the electron layer, the reflection coefficient $R^{2}$ as computed by the ray optics is unity for a friction-free layer. Any divergence from this theoretical value is interpreted as due to frictional absorption, and appropriate values of the 
collision frequency $\nu$ are affixed accordingly. Thus two out of the four observables are utilized for predicting the values of $N$ and $\nu$. The state of polarization as deduced by the ray optics is determined by the lowermost stratum with vanishingly small electron density. This prediction of the ray optics is contrary to actual experience.

The basic difficulties in the study of (1) are:

(a) Magneto-ionic anisotropy being of the cyclotonic class, separate wave equations describing the propagation of differently polarized waves cannot be set up unless $N=N_{0}$ (ray optics), or $\boldsymbol{\omega}_{H} \times \mathbf{k}=0$ (longitudinal propagation) or $\boldsymbol{\omega}_{H} \cdot \mathbf{k}=0$ (transverse propagation), $\mathbf{k}$ being the propagation vector.

(b) Magneto-ionic propagation in inhomogeneous media is described by equations of a class higher than those studied in other branches of mathematical physics.

The successive stages in the development of the general solution of (1) where the assumptions of the ray optics are not tenable can be arranged as follows:

(i) Removal of the partial time derivatives in (1) by applying Laplace-transform with respect to time and expressing the nonhomogeneous terms arising therefrom in terms of the prescribed initial conditions. Though the plasma frequency $\sqrt{N e^{2 / m \epsilon_{0}}}$ of the electron layer and the gyrofrequency $\left|e \mu_{0} \mathbf{H}_{e} / m\right|$ of the electrons around the terrestrial magnetic field $\mathbf{H}_{e}$ are of the same order as the frequencies of the incident waves, it can be shown that noncoherent scattering is not significant for the order of values of $N, \nu$, and $\omega_{H}$ inside the ionosphere. Hence the partial time derivatives in (1) can be replaced by the factor $i \omega=2 i \pi f$ without introducing any significant error.

(ii) A two-dimensional Laplace-transform with respect to the space-variables normal to $\boldsymbol{\nabla} N$ (on the assumption that $\boldsymbol{\nabla} N \times \boldsymbol{\nabla} \nu=0$ ) will reduce (1) into a pair of second-order ordinary linear differential equations, each containing two components of the electric vector (or its transform, if $\frac{\partial}{\partial t}$ is not replaced by $\left.i \omega\right)$ normal to $\boldsymbol{\nabla} N$. For vertical propagation of plane em waves this step is superfluous. In this latter case the equations controlling the propagation can be written as

$$
\begin{aligned}
& \frac{d^{2} E_{x}}{d z^{2}}+\left[K_{1}(z) E_{x}-i Q L(z) E_{y}\right]=0, \\
& \frac{d^{2} E_{y}}{d z^{2}}+\left[K_{2}(z) E_{y}+i Q L(z) E_{x}\right]=0,
\end{aligned}
$$

where $z$ is the vertical height and the functional dependence of $K_{1}(z), K_{2}(z)$, and $L(z)$ depends on the forms of $N(z)$ and $\nu(z)$.

(iii) The pair of equations in (2), for given forms of $N(z)$ and $\nu(z)$, can be studied by one of the following methods:

(a) Conversions into canonical form and application of the standard technique for the study of a system of equations [Ince, 1956].

(b) Application of perturbation methods to (2) or to equations derived from (2) and satisfied by suitable linear combinations of $E_{x}$ and $E_{y}$.

(c) Elimination of one of the dependent variables by raising the order of differentiation and studying the resulting fourth-order differential equation involving one dependent variable.

If utilized properly and interpreted correctly, all the above methods will lead to identical results within a certain degree of accuracy. However, the coefficient functions $K_{1}(z), K_{2}(z)$ and $L(z)$ are so complicated that even for the simplest types of electron layers, the solutions of (2) cannot be expressed in closed forms in terms of functions whose properties have already been studied. Fortunately, expressions for the physical observables $\tau, R^{2}$, $\mathscr{R}$, and $\Psi$ can be derived from the asymptotic solutions of (2). These solutions for $|z| \rightarrow \infty$ can always be expressed as

$$
\begin{aligned}
& E_{x} \sim \exp \left[i\left\{\omega t+\phi_{1 q}\right\}\right]+R_{q} \exp \left[i\left\{\omega t+\phi_{2 q}\right\}\right], \\
& E_{y} \sim \mathscr{R}_{1 q} \exp \left[i\left\{\omega t+\phi_{1 q}+\psi_{1 q}\right\}\right]+\mathscr{R}_{2 q} R_{q} \exp \left[i\left\{\omega t+\phi_{2 q}+\psi_{2 q}\right\}\right],
\end{aligned}
$$


where the suffix $q$ refers to the $q$ th magneto-ionic component, while $R_{q}, \mathscr{R}_{1 q, 2 q}, \phi_{1 q, 2 q}$, and $\psi_{1 q, 2 q}$ are real quantities expressed in terms of the layer parameters, pulsatance $\omega=2 \pi f$ of the incident wave, and the geomagnetic characteristics of the place of observation.

For each mode of propagation, the observables $\tau, R^{2}, \mathscr{R}$, and $\Psi$ are related to the above asymptotic solution as

$$
\tau=\left(\phi_{1 q} \sim \phi_{2 q}\right) / \omega, \quad R^{2}=\left[R_{q}\right]^{2}, \quad \mathscr{R}=\mathscr{R}_{1 q}, \quad \tan 2 \Psi=\frac{2 \mathscr{R}_{1 q}^{2}}{1-\mathscr{R}_{1 q}^{2}} \sin \psi_{1 q},
$$

where $\Psi$ is the tilt angle of the polarization ellipse traced out by the magnetic vector of the reflected echo.

For the transverse propagation the coupling coefficient $Q L(z)$ in (2) vanishes for any type of electron layer and separate equations describing the propagation of two linearly polarized waves can be set up. The transverse mode with the electric vector parallel to $\mathbf{H}_{e}$ is generally termed as the ordinary or $o$-mode, while the other termed as extraordinary or $e$-mode. For the longitudinal modes $K_{1}(z) \equiv K_{2}(z)=K(z)$ for any type of electron layer, and (2) reduces to

$$
\frac{d^{2}}{d z^{2}}\left(E_{x} \pm i E_{y}\right)+[K(z) \mp L(z)]\left(E_{x} \pm i E_{y}\right)=0 .
$$

These equations describe the propagation of two circularly polarized magneto-ionic components.

The effect of the steady magnetic field $\mathbf{H}_{e}$ on the propagation of the transverse $o$-mode or either of the longitudinal modes is nil or trivial. Hence these degenerate cases of propagation belong rather to the ionic than to the magneto-ionic class. Ionic propagation in linear, parabolic, and exponential electron layers has been studied by Epstein [1930; Hartree, 1931; Wilkes, 1940; Rydbeck, 1942; Wait, 1962], and others. Solutions of the wave equations for these cases can be expressed in terms of the functions of the confluent hypergeometric group, viz, Bessel or Weber functions.

The only remaining mode of these degenerate cases is the transverse $e$-mode whose propagation is characteristically influenced by the magnetic field $\mathbf{H}_{e}$. The propagation of this mode for the type of electron layers mentioned above is described by equations having, besides the essential singularity at infinity, more than one regular singularity in the finite region. This mode represents the simplest type of proper magneto-ionic propagation and has not been studied by any worker as yet. In part $\mathrm{I}$ of this paper the propagation of this transverse $e$-mode for a parabolic electron layer has been investigated in detail, and theoretical expressions for the relevant observables, viz, $\tau$ and $R^{2}$, have been derived in terms of the layer parameters.

As regards the general propagation with $\boldsymbol{\nabla} N \neq 0, \omega_{H} \times \mathbf{k} \neq 0, \omega_{H} \cdot \mathbf{k} \neq 0$, the functions $K_{1}(z)$, $K_{2}(z)$, and $L(z)$ in (2) are characterized by the presence of poles at the roots of the equation

$$
\frac{e^{2}}{m \epsilon_{0} \omega^{2}} \cdot N(z)=\frac{U(z)\left[U^{2}(z)-\omega^{2}\right]}{\left[U^{2}(z)-y_{L}^{2}\right]}
$$

where $U(z)=1-\frac{i \nu(z)}{\omega}$ and $y_{L}=\boldsymbol{\omega}_{H} \cdot \mathbf{k} / \omega$.

Hence the solutions of (2) have regular singularities at the roots of (6), which for $\nu=0$ occur within the layer unless $\omega<<\omega_{H}$ and the layer is very sharp. Behavior of the solutions of (2) depends on the number, location, and indices of these singularities. Any method developed for the study of the problem must preserve these basic features of the solutions of (2).

All the physical properties of inhomogeneous magneto-ionic media are cyclotonic dyads. Hence the matrices representing them cannot be diagonalized with all real elements. In other words, linear combinations of the type $\left[E_{x}+i \rho(z) E_{y}\right]$ or $\left[a_{1} E_{x}+b_{1} H_{x}+a_{2} E_{y}+b_{2} H_{y}\right]$ obeying separate (decoupled) wave equations do not exist for inhomogeneous magneto-ionic media. 
Försterling [1942], Rydbeck [1950], and lately Clemmow and Heading [1954], tried to determine suitable linear combinations of the horizontal components of the field vectors for which the coupling function would remain small throughout the layer to justify the application of perturbation methods. It can be shown that the coupling functions of these equations satisfied by the specific linear combinations suggested by these workers have precisely the same number and types of poles as $L(z)$ in (2) has. If it were not so, the solutions of the derived equations would differ radically from those of the original Maxwell-Lorentz equations.

A second-order linear differential equation is basically equivalent to a pair of coupled firstorder equations; moreover, irrespective of the nature of the coefficient functions, a first-order equation can always be expressed in closed integral form, while a second-order equation cannot be so easily integrated unless the coefficients are elementary functions of the independent variable. Hence the methods adopted by these workers amount, at least in principle to, the evaluation of the solutions, in closed forms, for any linear differential equation.

Besides the above-mentioned attempts and the recently developed methods of numerical integration [Budden, 1955; Barron and Budden, 1959], this complicated problem has not, as yet, been tackled by any worker. In part II of this paper the pair of equations (2) for a parabolic electron layer has been investigated, and theoretical expressions for the four observables mentioned earlier have been evaluated from the relevant asymptotic solutions.

\section{Wave Equations for the Transverse Modes}

Consider the propagation of a continuous train of em waves of frequency $f=\omega / 2 \pi$ in a magnetoionic medium with the following assumptions:

(i) The propagation is along the vertical $z$-axis;

(ii) The steady magnetic field $\mathbf{H}_{e}$ is along the $x$-axis;

(iii) Noncoherent scattering is absent;

(iv) Collision damping $\nu$ is independent of $z$; and

(v) The electron-density $N(z)$ is of the form

$$
\begin{aligned}
N(z) & =N_{m}\left(1-\frac{z^{2}}{l^{2}}\right) \text { for }-l \leqslant z \leqslant l, \\
& =0 \text { for } z^{2}>l^{2} .
\end{aligned}
$$

It can be shown that (1), under the above set of conditions, reduces to

$$
\begin{gathered}
\frac{d^{2} E_{x}}{d z^{2}}+K^{2}\left[z^{2}-\frac{a^{2}+b^{2}}{2}\right] E_{x}=0, \\
\frac{d^{2} E_{y}}{d z^{2}}+K^{2}\left[\frac{\left(z^{2}-a^{2}\right)\left(z^{2}-b^{2}\right)}{\left(\mathrm{z}^{2}-z_{0}^{2}\right)}\right] E_{y}=0,
\end{gathered}
$$

where

$$
K^{2}=4 \pi^{2} h_{1}^{2} / l^{4}, \quad h_{1}^{2}=l^{2} /(1-i Z) \lambda_{m}^{2}, \quad Z=\nu / \omega,
$$

$\lambda_{m}=\frac{2 \pi c}{\omega_{m}}$, the wavelength in vacuum corresponding to the maximum plasma-frequency of the layer,

$$
\begin{aligned}
& \omega_{m}^{2}=\frac{e^{2} N_{m}}{m \epsilon_{0}}, \quad \omega_{H}^{2}=\left[\mu_{0} e \mathbf{H}_{e} / m\right]^{2} . \quad c=3.10^{8} \mathrm{~m} / \mathrm{s}, \\
& a^{2}=\left(1-h_{2}^{2}+h_{2} h_{3}\right) l^{2}, \quad b^{2}=\left(1-h_{2}^{2}-h_{2} h_{3}\right) l^{2}, \quad z_{0}^{2}=\left(1-h_{2}^{2}+h_{3}^{2}\right) l^{2}, \\
& h_{2}^{2}=\frac{\omega^{2}(1-i Z)}{\omega_{m}^{2}}, \quad h_{3}^{2}=\frac{\omega^{2}}{(1-i Z) \omega_{m}^{2}} .
\end{aligned}
$$


Equation (8) describe the transverse $o$-mode for parabolic layers. The magnetic field $\mathbf{H}_{e}$ has no effect on this mode. This equation has been discussed in details by Rydbeck [1942].

Equation (9) describing the transverse $e$-mode represents the simplest type of magneto-ionic propagation in a parabolic layer. It is characterized by the pair of regular singularities at $z= \pm z_{0}$. The nature of propagation for $-z_{0} \leqslant z \leqslant z_{0}$ (the magneto-ionic core) is entirely different from that in the regions $z^{2}>z_{0}^{2}$ (fringes of the layer). Evidently (9) reduces to (8) for $\omega_{H}=0$. For $\omega^{2} \sim \omega_{m}^{2}$ $\pm \omega_{H}^{2}$, solutions of (9) near $z \rightarrow-l$ (bottom of the layer) can be represented accurately by the asymptotic forms.

\section{Solutions of the Wave Equation}

A quadratic transformation of the independent variable in (9) results in an equation having one elementary singluarity (exponent difference $\frac{1}{2}$ ) at the origin, one regular singularity at $z_{0}^{2}$ and an essential one of grade 1 at $\infty$. Equation (9), or the one transformed as indicated above, belongs to a class higher than the confluent hypergeometric equations (Whittaker, Weber, and Bessel equations) which accommodate one regular and one essential singularity, or the Mathieu class having besides the essential one, a pair of elementary singularities.

Changing the variables from $z$ to $\xi=\left(1-z^{2} / z_{0}^{2}\right)$ and from $E_{y}(z)$ to $F(\xi)=e^{-K\left(z^{2}-z_{0}^{2}\right) / 2} \cdot E_{y}(z),(9)$ can be written as

$$
\xi(1-\xi) \frac{d^{2} F}{d \xi^{2}}+\left[-\xi_{0} \xi^{2}+\left(\xi_{0}-\frac{1}{2}\right) \xi\right] \frac{d F}{d \xi}+\left[M \xi_{0} \xi-\Lambda\right] F=0,
$$

where

$$
\begin{aligned}
& \xi_{0}=i K z_{0}^{2}=2 i \pi h_{1}\left(1-h_{2}^{2}+h_{3}^{2}\right), \\
& M=\frac{i K}{4}\left[a^{2}+b^{2}-z_{0}^{2}\right]-\frac{1}{4}=\frac{i \pi h_{1}}{2}\left[1-h_{2}^{2}-h_{3}^{2}\right]-\frac{1}{4}, \\
& \Lambda=\frac{K^{2}}{4}\left(z_{0}^{2}-a^{2}\right)\left(z_{0}^{2}-b^{2}\right)=-\pi^{2} h_{1}^{2} h_{3}^{2}\left(h_{2}^{2}-h_{3}^{2}\right) .
\end{aligned}
$$

Consider the integral

$$
\int_{C}(\eta-\xi)^{M} \theta(\eta) d \eta
$$

where $\theta(\eta)$ obeys the equation

$$
\frac{d}{d \eta}\left[\eta(1-\eta) \frac{d}{d \eta}\left(\eta^{M} \theta\right)+\left\{-\xi_{0} \eta^{2}+\left(\xi_{0}-\frac{1}{2}\right) \eta-M\right\} \eta^{M} \theta\right]-\Lambda \eta^{M} \theta=0
$$

Solutions of (1la) can be written as

$$
\begin{aligned}
\theta(\eta) & =\eta^{-M}(1-\eta)^{-M-1 / 2} e-\eta \xi_{0} \theta,(\eta), \\
\theta_{1}(\eta) & =1-\frac{M\left(M-\frac{1}{2}-\xi_{0}\right)+\Lambda}{M-1} \cdot \eta+\text { higher powers of } \eta, \\
& =1-\frac{M\left(M-\frac{1}{2}\right)+\Lambda}{M-\frac{1}{2}} \cdot(1-\eta)+\text { higher powers of }(1-\eta) .
\end{aligned}
$$


Since the series $\theta_{1}(\eta)$ is analytic throughout the finite range of $\eta$, the behavior of $\theta(\eta)$ near the singularities 0,1 , and $\infty$ are as follows:

$$
\begin{aligned}
\theta(\eta) & \rightarrow \eta^{-M} \text { as } \eta \rightarrow 0, \\
& \rightarrow(1-\eta)^{-M-1 / 2} \text { as } \eta \rightarrow 1, \\
& \rightarrow e^{-\eta \xi_{0}} \text { as } \eta \rightarrow \infty
\end{aligned}
$$

Integral (11) satisfies (10), provided the contour $C$ is so chosen that the bilinear concomitant of $(\eta-\xi)^{M}$ and $\theta(\eta)$ vanishes at the extremities of $C$. From the above behavior of $\theta(\eta)$ near its singularities, contours may be selected for different ranges of $\xi$ such that (11) identically satisfies (10) (see table 1).

TABLE 1. Contours for different ranges of $\xi$ so that (11) identically satisfies (10)

\begin{tabular}{c|r|l}
\hline \multicolumn{1}{c|}{ Range } & \multicolumn{1}{|c}{ Contour $C$} & \multicolumn{1}{|c}{ Solution } \\
\hline & (i) $\eta=1$ to $\eta=\infty, R_{e} \xi_{0}>0$ & $F_{1}(\xi)$ \\
$0<R_{e} \xi \leqslant 1$, & (ii) $\eta=0$ to $\eta=-\infty, R_{e} \xi_{0}<0$ & $F_{2}(\xi)$ \\
$z^{2} \leqslant\left|z_{0}^{2}\right| ;$ & $F_{3}(\xi)$ \\
Magneto-ionic & (iii) $\eta=0$ to $\eta=\xi$ & $F_{4}(\xi)$ \\
core & (iv) $\eta=\xi$ to $\eta=1$ & $F_{5}(\xi)=F_{1}(\xi)$ \\
\hline$\infty<R_{e} \xi \leqslant 0$, & (v) $\eta=1$ to $\eta=\infty, R_{e} \xi_{0}>0$ & $F_{6}(\xi)$ \\
$z^{2}>\left|z_{0}^{2}\right| ;$ & (vi) $\eta=\xi$ to $\eta=-\infty, R_{e} \xi_{0}<0$ & $F_{7}(\xi)$ \\
Magneto-ionic & (vii) $\eta=\xi$ to $\eta=0$ & $F_{8}(\xi)$ \\
fringe. & (viii) $\eta=0$ to $\eta=1$ & \\
\hline
\end{tabular}

The general solution inside the core can be expressed by selecting the contour $C$ as consisting of a line from $\eta=0$ to $\eta=\xi$, a semicircle around $\eta=\xi$, and a line from $\eta=\xi$ to $\eta=1$. Carrying out the integrations along the different branches of $C$ and introducing the time factor $e^{i \omega t}$, the general solution inside the magneto-ionic core can be expressed in terms of the original variable as

$$
E_{y}(z ; t)=f_{1}\left(1-\frac{z^{2}}{z_{0}^{2}}\right) \exp \left[i\left\{\omega t-\frac{K}{2} z^{2}\right\}\right]+P f_{2}\left(\frac{z}{z_{0}}\right) \exp \left[i\left\{\omega t+\frac{K}{2} z^{2}\right\}\right]
$$

where $f_{1}\left(1-z^{2} / z_{0}^{2}\right)=\sum_{0}^{\infty} a_{n}\left(1-z^{2} / z_{0}^{2}\right)^{n+1}$,

$$
\begin{aligned}
& f_{2}\left(z / z_{0}\right)=\sum_{0}^{\infty} b_{n}\left(z / z_{0}\right)^{2 n+1}, \\
& a_{0}=b_{0}=1, \quad P=\frac{2 \Gamma\left(\frac{1}{2}-M\right)}{\sqrt{\pi} \Gamma(1-M)} \cdot \exp \left[-i\left\{2 \pi M+K z_{0}^{2}\right\}\right], \\
& a_{1}=\left(\Lambda+\frac{1}{2}-\xi_{0}\right) / 2, \quad b_{1}=\left[\Lambda-(M+1) \xi_{0}\right] /(3 / 2), \\
& (n+1)(n+2) a_{n+2}-\left[n^{2}-n\left(\xi_{0}-\frac{3}{2}\right)+\Lambda+\frac{1}{2}-\xi_{0}\right] a_{n+1}+(M-n) a_{n}=0, \\
& (n+1)\left(n+\frac{5}{2}\right) b_{n+2}-\left[n^{2}-n\left(\xi_{0}-\frac{5}{2}\right)+\Lambda+\frac{3}{2}-\xi_{0}\right] b_{n+1}-\xi_{0}(M+n+1) b_{n}=0 .
\end{aligned}
$$

The right-hand side of (12) represents two wavy disturbances of complex amplitude ratio $P$, 
moving in opposite directions with complex phase factors $\left(-K z^{2} / 2-i \ln f_{1}\right)$ and $\left(K z^{2} / 2-i \ln f_{2}\right)$, respectively. For very long waves such that $\omega<\omega_{H}$, the location of the singularity at $z_{0}^{2}$ with $\nu=0$ is outside the physical layer of half-width $l$. Hence for such waves, the composite disturbance at the bottom of the layer $z=-l$ can be expressed as

$$
E_{y}(-l ; t)=\left[1+\frac{f_{2}(-l)}{P f_{1}(-l)} \exp \left(i K l^{2}\right)\right] E_{y}^{0}(-l ; t),
$$

where $E_{y}^{0}(-l ; t)$ is the state of the wave incident on the layer. If

$$
R_{e} e^{-i \phi_{e}}=\frac{f_{2}(-l) \exp \left(i K l^{2}\right)}{P f_{1}(-l)}
$$

where $R_{e}$ and $\phi_{e}$ are real quantities, the physical observables are related to the above variables as: $R_{e}$ is the amplitude of the downcoming wave at $z=-l$, relative to that of the upgoing wave at that point $=\bar{R}_{e} e^{-\chi_{e}}$, where $\bar{R}_{e}$ is the reflection coefficient for a collision free layer, and $\chi_{e}$ is the total absorption in the layer due to collisional damping. If $\tau_{e}$ be the time delay observed on the CRO screen and $h_{b}$ the height of the bottom layer from the transmitter-receiver,

$$
\tau_{e}=\frac{2 h_{b}}{c}+\frac{\phi_{e}}{\omega}
$$

where $c=3.10^{8} \mathrm{~m} / \mathrm{sec}$. Hence solution (12) of (9) furnishes information about all the observables of the transverse $e$-mode, those of the $o$-mode being derivable from these results of putting $\omega_{H}=0$.

\section{Asymptotic Solutions}

For waves used in normal radio cummunications, $\omega_{H} \ll \omega \sim \omega_{m}$ and hence $\left|z_{0}^{2}\right| / l^{2} \rightarrow 0$. Solutions of (9) for these frequency bands are given by the asymptotic behavior of $E_{y}(z ; t)$. In this region of frequencies consider the integral

$$
\int_{C} e^{-\eta \xi_{0} \xi} v(\eta) d \eta
$$

where

$$
\eta(1-\eta) \frac{d^{2} v}{d \eta_{2}}+\left[\xi_{0} \eta^{2}\left(\xi_{0}+\frac{7}{2}\right) \eta+(M+2)\right] \frac{d v}{d \eta}+\left[2 \xi_{0} \eta-\left(\xi_{0}+\Lambda+\frac{3}{2}\right)\right] v=0
$$

Solutions of (14a) can be expressed as

$$
v(\eta)=\eta^{-(1+M)}(1-\eta)^{M-1 / 2} e^{\eta \xi_{0}} \sum_{0}^{\infty} \gamma_{n} \eta^{\eta}
$$

with $\gamma_{0}=1$,

$$
\begin{aligned}
& \gamma_{1}=-\frac{\Lambda}{M}, \text { and } \\
& (n+2)(n+1-M) \gamma_{n+2}+\left[(n+1)\left(n+\frac{1}{2}-\xi_{0}\right)+\Lambda\right] \gamma_{n+1}-n \xi_{0} \gamma_{n}=0 .
\end{aligned}
$$

Integral (14) will satisfy (10) if the contour $C$ is properly chosen. Since the real axis in the $\eta$-plane is the cut plane of the integral in (14), the solution for the sector $0 \leqslant \arg \left(-\xi_{0} \xi\right)<\pi$ will be different from the solution in the sector $\pi \leqslant \arg \left(-\xi_{0} \xi\right)<2 \pi$. For $\left(-\xi_{0} \xi\right)=\left|\xi_{0} \xi\right| e^{i \alpha}$ and $R_{e} M<0$, 
the contour $C$ may be chosen to consist of

(a) a line $C_{1}$, joining $\eta=0$ to $\eta=-|r| e^{-i \alpha}$,

(b) a line $C_{2}$, joining $\eta=-|r| e^{-i \alpha}$ to $\eta=1-|r| e^{-i \alpha}$, and

(c) a loop $C_{3}$, in the positive direction round $\eta=1$ starting and ending at $\eta=1-|\gamma| e^{-i \alpha}$.

As $|\gamma| \rightarrow \infty$, the contribution from $C_{2}$ will vanish, and the solution of (10) for $\xi \rightarrow-\infty$ can be expressed as

$$
\begin{aligned}
F(\xi)=\int_{0}^{-\infty e^{-i \alpha}} e^{-\eta \xi_{0} \xi} \cdot \eta^{-(1+M)}\left[\sum_{0}^{\infty}(1-\eta)^{M-1 / 2} e^{\eta \xi_{0}} \cdot \gamma_{n} \eta^{n}\right] d \eta & \\
& +\int_{1-\infty e^{-i \alpha}}^{(1+)} e^{-\eta \xi_{0} \xi}(1-\eta)^{M-1 / 2}\left[\sum_{0}^{\infty} \eta^{-(1+M)} e^{\eta \xi} \gamma_{n} \eta^{n}\right] d \eta .
\end{aligned}
$$

For $|\xi| \gg 1$, the asymptotic solution can be obtained by putting $\eta=\left(u_{1} / \xi_{0} \xi\right)$ in the first integral and $1-\eta=\left(-u_{2} / \xi_{0} \xi\right)$ in the second. If further, $\left(1 / \xi_{0} \xi\right)$ is written as $e^{i \pi} /\left(-\xi_{0} \xi\right)$ for $0<\alpha \leqslant \pi$ and as $e^{-i \pi} /\left(-\xi_{0} \xi\right)$ for $\pi<\alpha \leqslant 2 \pi$, the form of the solution as $z \rightarrow-l$ can be expressed as

$E_{y}(\xi) \sim \Gamma(-M) \cdot\left(-\xi_{0} \xi\right)^{M} \cdot V_{1}\left(-\xi_{0} \xi\right) \cdot \exp \cdot\left[1 / 2 \xi_{0} \xi\right]$

$$
+\frac{2 \pi}{\Gamma(-M+1 / 2)} \cdot\left(-\xi_{0} \xi\right)^{-M-1 / 2} V_{2}\left(-\xi_{0} \xi\right) \exp \cdot\left[-1 / 2 \xi_{0} \xi\right]
$$

where

$$
\begin{aligned}
& V_{1}\left(-\xi_{0} \xi\right)=1+\sum_{1}^{\infty} \frac{M(M-1) \ldots(M-n+1)}{n !\left(\xi_{0} \xi\right)^{n}} \cdot \alpha_{n}, \\
& V_{2}\left(-\xi_{0} \xi\right)=1+\sum_{1}^{\infty}(-1)^{n} \cdot \frac{\left(M+\frac{1}{2}\right)\left(M-\frac{1}{2}\right) \ldots\left(M+\frac{1}{2}-n+1\right)}{n !\left(\xi_{0} \xi\right)^{n}} \beta_{n},
\end{aligned}
$$

and

$$
\begin{aligned}
& \Sigma \alpha_{n} x^{n} \equiv \sum_{0}^{\infty} \gamma_{n}\left[x^{n}(1-x)^{M-1 / 2} \cdot e^{x \xi_{0}}\right], \\
& \Sigma \beta_{n} x^{n} \equiv \sum_{0}^{\infty} \gamma_{n}\left[(1-x)^{n-1-M} \cdot e^{(1-x) \xi_{0}}\right] .
\end{aligned}
$$

Introducing the time factor $e^{i \omega t}$ and passing on to the original variables, the form of the disturbance as $z \rightarrow-l$ for $\omega_{H} \ll \omega \sim \omega_{m}$ can be expressed as

$$
E_{y}(z ; t) \sim f_{3}(z) \exp \cdot\left[i\left\{\omega t-\frac{K z^{2}}{2}\right\}\right]+f_{4}(z) \exp \cdot\left[i\left\{\omega t+\frac{K z^{2}}{2}\right\}\right]
$$

where

$$
\begin{aligned}
& f_{3}(z) \sim \frac{2 \pi\left[i K\left(z^{2}-z_{0}^{2}\right)\right]^{-M-1 / 2}}{\Gamma\left(-M+\frac{1}{2}\right)} \exp \left[-i\left\{\frac{K z_{0}^{2}}{2}+\pi \bar{M}-\frac{\Lambda-i \bar{M}\left(i \bar{M}-\xi_{0}\right)}{K\left(z^{2}-z_{0}^{2}\right)}\right\}\right], \\
& f_{4}(z) \sim \Gamma(-M)\left[i K\left(z^{2}-z_{0}^{2}\right)\right]^{M} \exp \left[i\left\{\frac{K z_{0}^{2}}{2}+\pi \bar{M}-\frac{\Lambda-i \bar{M}\left(i \bar{M}-\xi_{0}\right)}{K\left(z^{2}-z_{0}^{2}\right)}\right\}\right] \\
& i \bar{M}=M+1 / 4
\end{aligned}
$$

Since $z<0$ near the bottom of the layer, the first term represents the upgoing (incident) wave while the other represents the downcoming or reflected wave. Hence if $\boldsymbol{E}_{y}^{0}(-l ; t)$ represents the state of the incident wave at $z=-l$, the composite disturbance at that point can be written as

$$
E_{y}(-l ; t)=\left[1+R_{e} e^{-i \phi_{e}}\right] E_{y}^{0}(-l ; t)
$$


where

$$
R_{e} e^{-i \phi_{e}}=\left|f_{4}(z) \exp \left(i K z^{2}\right) / f_{3}(z)\right|_{z=-l}
$$

For the $o$-mode, given by (8), similar expressions can be deduced from above by letting $\omega_{H} \rightarrow 0$.

If $\bar{R}_{0}^{2}$ and $\bar{R}_{e}^{2}$ be the reflection coefficients of the two modes in the absence of collisional damping, it can be shown that

$$
\begin{gathered}
\frac{c}{\pi l \omega_{m}} \ln \left[\frac{\bar{R}_{0}^{2}}{1-\bar{R}_{0}^{2}}\right]=f_{0}=1-\frac{\omega^{2}}{\omega_{m}^{2}}, \\
\frac{c}{\pi l \omega_{m}} \ln \left[\frac{\bar{R}_{e}^{2}}{1-\bar{R}_{e}^{2}}\right]=f_{e}=1-\frac{\omega^{2}}{\omega_{m}^{2}}-\frac{\omega_{H}^{2}}{\omega_{m}^{2}} .
\end{gathered}
$$

Here $\bar{R}_{0}^{2}$ and $\bar{R}_{e}^{2}$ are unity throughout the whole range of frequencies except at the critically penetrating bands ( $\omega^{2} \approx \omega_{m}^{2}$ for $o$-mode and $\omega^{2} \approx \omega_{m}^{2}-\omega_{H}^{2}$ for the e-mode), where they suddenly fall to half the value. Variations of the left-hand expressions in (16a) and (16b) with $h_{2}=\frac{\omega}{\omega_{m}}$ have been shown in figure 1 .

The total absorptions $\chi_{0}$ and $\chi_{e}$ for the two modes are given by

$$
\chi_{0}=\ln \left(\bar{R}_{0} / R_{0}\right) \text { and } \chi_{e}=\ln \left(\bar{R}_{e} / R_{e}\right) .
$$

Separating the right-hand side of (15a) into real and imaginary parts and retaining only the dominant terms, it can be shown that

$$
\begin{aligned}
& \chi_{0} \sim \frac{g \nu}{2}\left[\left\{1-\frac{f_{0}}{2}\right\} \ln \left(\frac{4}{f_{0}}\right)-1\right], \\
& \chi_{e} \sim \frac{g \nu}{2}\left[\left\{1-\frac{f_{e}}{2}\right\} \ln \left(\frac{4}{f_{e}}\right)-1\right], \\
& g=\frac{\omega_{m}}{\omega} \cdot \frac{l}{c} .
\end{aligned}
$$

Figure 2 shows the variations of $\chi_{0}$ and $\chi_{e}$ with $h_{2}=\omega / \omega_{m}$ (for $\nu=0$ ).

If $\tau_{0}$ and $\tau_{e}$ be the observed time-delays between the first reflected waves of the two modes and the ground pulse it can be shown that

$$
\begin{gathered}
\tau_{0} \sim \frac{2 h_{b}}{c}+g\left[1-\frac{f_{0}}{2}-\frac{f_{0}}{2} \ln \left(\frac{4}{f_{0}}\right)\right]-\frac{\pi}{\omega}, \\
\tau_{e} \sim \frac{2 h_{b}}{c}+g\left[1-\frac{f_{e}}{2}-\frac{f_{e}}{2} \ln \left(\frac{4}{f_{e}}\right)\right]-\frac{\pi}{\omega},
\end{gathered}
$$

where $h_{b}=$ height of the bottom of the layer. Variations of the function $y=\frac{1}{2} \tau-\frac{h_{b}}{c}+\frac{\pi}{2 \omega}$ with with $h_{2}=\frac{\omega}{\omega_{m}}$ (for $\nu=0$ ) for the two modes have been shown in figure 3 .
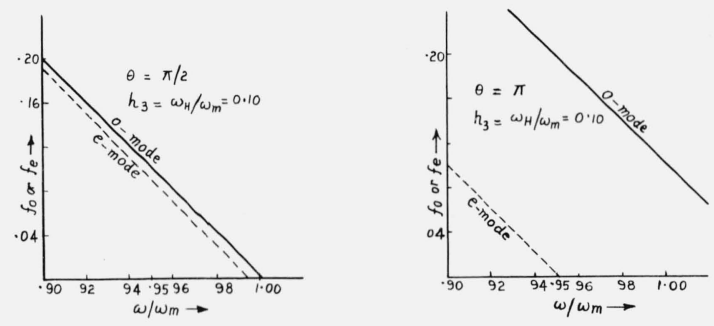

FiguRE 1. Reflection coefficient as a function of frequency.
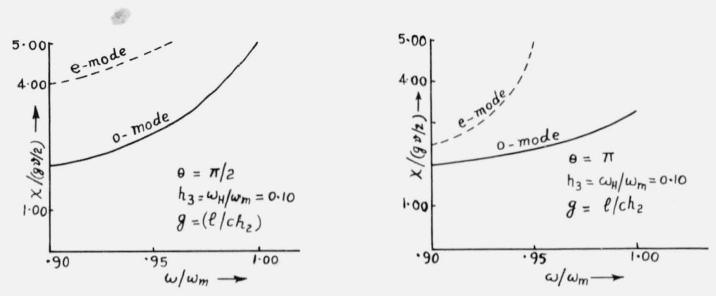

FIGURE 2. Absorption coefficient as a function of frequency. 

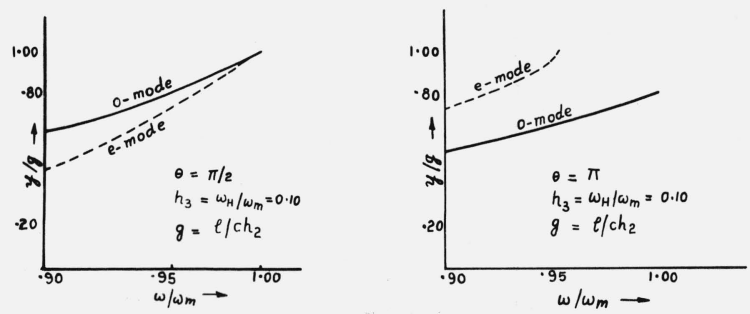

Figure 3. Time-delay as a function of frequency.

Expressions (16a) to (18b) furnish informations about all the quantities observed in the radio exploration of the ionosphere. Though the above analysis has been carried out only for a parabolic layer, the method can be extended to any other type of electron layer.

\section{Appendix. List of Symbols and Âbbreviations}

Electric vector of the wave $\mathbf{E}(x, y, z ; t)$.

Permittivity of free space. $\epsilon_{0}$.

Permeability of free space. $\mu_{0}$.

Steady magnetic field.... $\mathbf{H}_{e}$.

Collision frequency.... $\nu$.

Electronic mass and charge $m, e$.

Frequency of the traveling wave. $f=\omega / 2 \pi$.

Velocity of light c.

Electron density... $N$.

Maximum electron density.... $N_{m}$.

Half-width of the layer. l.

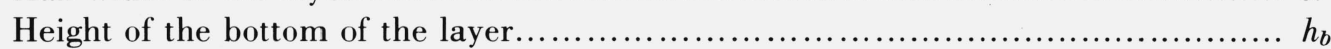

Reflection coefficients. $\bar{R}_{o}^{2}, \bar{R}_{e}^{2}$

Total absorption... $\chi_{0}, \chi_{e}$

Observed time delay on the CRO screen. $\tau_{0}, \tau_{e}$

Ratio of the axes of the polarization ellipse TR.

Tilt of the polarization ellipse $\Psi$.

Abbreviations:

$$
\begin{aligned}
& \omega_{m}^{2}=N_{m} e^{2} / m \epsilon_{0}, \quad \lambda_{m}=2 \pi c / \omega_{m}, \quad \omega_{H}=\left|e \mu_{0} \mathbf{H}_{e} / m\right|, \\
& Z=1-i \nu / \omega, \quad K^{2}=4 \pi^{2} h_{1}^{2} / l^{4}=\left(\frac{\omega_{m}}{l c}\right)^{2} /(1-i Z), \\
& h_{1}^{2}=l^{2} /(1-i Z) \lambda_{m}^{2}, \quad h_{2}^{2}=(1-i Z) \omega^{2} / \omega_{m}^{2}, \quad h_{3}^{2}=\omega_{H}^{2} /(1-i Z) \omega_{m}^{2}, \\
& a^{2}=\left(1-h_{2}^{2}+h_{2} h_{3}\right) l^{2}, \quad b^{2}=\left(1-h_{2}^{2}-h_{2} h_{3}\right) l^{2}, \quad z_{0}^{2}=\left(1-h_{2}^{2}+h_{3}^{2}\right) l^{2}, \\
& M=\frac{i \pi h_{1}}{2}\left(1-h_{2}^{2}-h_{3}^{2}\right)-\frac{1}{4}=i \bar{M}-\frac{1}{4}, \\
& \Lambda=-\pi^{2} h_{1}^{2} h_{3}^{2}\left(h_{2}^{2}-h_{3}^{2}\right), \\
& \xi_{0}=i K z_{0}^{2}, \quad g=l / c h_{2}(\text { for } \nu=0), \\
& f_{0}=1-\frac{\omega^{2}}{\omega_{m}^{2}}, \quad f_{e}=1-\frac{\omega^{2}}{\omega_{m}^{2}}-\frac{\omega_{H}^{2}}{\omega_{m}^{2}} .
\end{aligned}
$$




\section{References}

Appleton, E. V. (1927), Magnetoionic propagation, U.R.S.I. Reports, Washington.

Barron, D. W., and K. G. Budden (1959), The numerical solution of differential equations governing the reflexion of long radio waves from the ionosphere, III, Proc. Roy. Soc. London, Ser. A 249, 387-401.

Budden, K. G. (1955), The numerical solution of differential equations governing reflexion of long radio waves from the ionosphere, Proc. Roy. Soc. London, Ser. A 227, 516-537.

Clemmow, P. C., and J. Heading (1954), Coupled forms of the differential equations governing radio propagation in the ionosphere, Proc. Cambridge Phil. Soc. 50, 319.

Epstein, P. S. (1930), Reflection of waves in an inhomogeneous absorbing medium, Proc. Natl. Acad. Sci. U.S. 16, $627-637$.

Forsterling, K. (1942), Uber die Ausbreitung elektro-magnetischer Wellen in einem magnetisierten Medium bei senkrechter Incidenz, Hochfrequenztech. u. Elektroakust. 59, 10-22.

Hartree, D. R. (1929), The propagation of electromagnetic waves in a stratified medium, Proc. Cambridge Phil. Soc. 25, 97-120.

Hartree, D. R. (1931), Optical and equivalent paths in a stratified medium treated from a wave standpoint, Proc. Roy. Soc. London, Ser. A 131, 428-450.

Ince, E. L. (1956), Ordinary Differential Equations, ch. XIX, p. 467 (Dover Publications, New York, N.Y.).

Rydbeck, O. E. H. (1942), The reflection of electromagnetic waves from a parabolic friction-free ionized layer, J. Appl. Phys. 13, 577-581.

Rydbeck, O. E. H. (1950), Magnetoionic triple splitting of ionospheric waves, J. Appl. Phys. 21, 1205-1214.

Wait, J. R. (1962), Electromagnetic Waves in Stratified Media (Pergamon Press, Oxford).

Wilkes, M. V. (1940), The theory of reflection of very long wireless waves from the ionosphere, Proc. Roy. Soc. London, Ser. A 1 75, 143-163.

(Paper 69D9-563) 